\title{
MIGRACIONES EXTREMEÑAS EN EL UMBRAL DEL SIGLO XXI: LA INMIGRACIÓN DE RETORNO
}

\author{
Antonio Pérez Díaz \\ Felipe Leco Berrocal \\ Departamento de Arte y Ciencias del Territorio. Universidad de Extremadura \\ aperez@unex.es, fleco@unex.es
}

\section{RESUMEN}

La emigración ha constituido uno de los acontecimientos de mayor relevancia demográfica, social, económica y política en Extremadura. El volumen de extremeños que continuaba residiendo fuera de Extremadura en 2010 se situaba en 608.101 personas, lo que representa el 54,9 por 100 del total de población censada en la región y un 63,9 por 100 de los nacidos y residentes en el territorio regional. Este colectivo ha venido nutriendo una inmigración de retorno que, desde mediados de los años setenta, ha experimentado cambios significativos tanto en su volumen como en su composición interna. La consolidación de nuevos patrones de retorno desde la segunda mitad de los años ochenta y las características de esta corriente en la primera década del siglo XXI, constituyen el objetivo central de este trabajo.

Palabras clave: Inmigración, retorno, dinámica espacial, estructura demográfica.

\section{ABSTRACT}

Emigration has been one of the most relevant demographic, social, economic and political events in Extremadura. The number of people from Extremadura who continued to live outside of the region in 2010 is 608.101 , representing $54,9 \%$ of the total population, according to the regional census, and $63,9 \%$ of those born and residing in the region. This total has been feeding a return immigration that, since the middle of the seventies, has experienced significant changes, not only in numbers, but also in its internal composition. The consolidation of new return patterns since the second half of the eighties and the

Fecha de recepción: mayo 2011.

Fecha de aceptación: diciembre 2012. 
characteristics of this flow during the first decade of the 21 st century are the main focus of this study.

Key words: Immigration, return, spatial dynamics, demographic structure.

\section{INTRODUCCIÓN}

La emigración ha sido considerada como el fenómeno histórico de mayor relevancia en la Extremadura del siglo XX y, por ello, como uno de los elementos definitorios de la historia, del presente y del futuro del pueblo extremeño ${ }^{1}$. Aunque aparentemente exagerada, esta apreciación adquiere consistencia cuando se estima que fueron en torno a ochocientos mil los emigrantes netos que registró Extremadura entre finales de los años cincuenta y mediados de los setenta. No en vano, en 1987, extinguida ya la corriente emigratoria de la etapa anterior, aún ascendía a 729.532 el número de extremeños que residía en territorio nacional fuera de su región de nacimiento.

Los destinos preferentes de aquella emigración masiva y familiar fueron las periferias metropolitanas de las áreas industrializadas. Móstoles o Alcorcón en Madrid, Barakaldo o Santurtzi en Vizcaya, Sant Boi de Llobregat o Sabadell en Barcelona y Alaquás o Mislata en Valencia, se poblaron de colonias de extremeños que respondían al efecto llamada de los emigrantes más madrugadores. El otro destino fue Europa Occidental, preferentemente Alemania Federal, Francia y Suiza. Aunque la evaluación de esta corriente externa se ve dificultada por la parcialidad de los datos del Instituto Español de Emigración, se estima que debió afectar a más de 56.300 extremeños (Cayetano, 1996).

Pero la crisis económica provocó la aparición del paro industrial en los primeros setenta y, con ello, el estrangulamiento de la corriente emigratoria. Los que emigraron en los cincuenta y sesenta comenzaban a experimentar cierta dosis de insatisfacción. Habían envejecido y se habían vuelto más nostálgicos. Comenzaban a imponerse pautas de consumo de segunda residencia y, muchos de ellos, aún poseían una casa en su pueblo. Surgía así, en la segunda mitad de los setenta y con una cierta intensidad, el fenómeno del retorno, que habría de incrementarse y experimentar importantes transformaciones estructurales en los años siguientes.

Se llegaba así a una situación insólita: por primera vez en todo el siglo XX, los saldos migratorios mostraban signo positivo en el quinquenio que separa el Censo de 1981 del Padrón de 1986. Todos los indicios aventuraban un cambio sustancial en el comportamiento demográfico de la región. Se magnificó el fenómeno retorno y se identificó con el síntoma de la bonanza económica y las reformas sociales.

Poco tiempo tuvo que transcurrir para que se evidenciara, una vez más, la fragilidad de las previsiones demográficas. Las distintas variables de comportamiento biológico, merced al papel homogenizador de una información cada vez más universal, habían seguido los derroteros comunes al conjunto del territorio nacional, en la órbita europea. La fecundidad, que había superado la media, se aproximó, llegando a igualarse, en una tendencia descendente.

1 Ley 3/1986 de 24 de mayo, de la Extremeñidad. DOE núm. 53, de 26 de junio de 1986. 
En consecuencia, el envejecimiento ha seguido aumentando, siendo previsible un escenario más agudo en los años próximos, sin que ello quiera decir que esta sea una perspectiva invariable. La población absoluta se ha estabilizado en umbrales que oscilan poco alrededor del crecimiento cero. Por último, no sólo no se ha cerrado el bucle, sino que la población extremeña parece haberse estancado en un nuevo óptimo demográfico de larga duración, definiendo el nuevo papel de la región en el contexto nacional. Los síntomas de cambio no eran sino el exponente de una coyuntura general en la que los factores económicos exteriores se impusieron a la dinámica interior, tal como ha venido ocurriendo en tantas otras ocasiones.

\section{OBJETIVOS Y FUENTES}

Desde que, a mediados de los años setenta y como consecuencia de la crisis que afectó a las economías occidentales, se produjera una profunda alteración en la dinámica espacial de los españoles, la emigración extremeña ha fluctuado al dictado de las cambiantes coyunturas económicas, ha visto alterarse sustancialmente sus perfiles estructurales y, en no pocas ocasiones, ha llegado a verse superada por la potencia del flujo inmigratorio. Nunca, sin embargo, ha mostrado síntomas de un agotamiento absoluto: entre los años 1991 y 2009, la cifra registrada en Extremadura ha ascendido a 220.486 emigrantes, lo que arroja un promedio anual superior a las 11.604 bajas. No es de extrañar, en consecuencia, que la actualización del Padrón Municipal de 2010 arrojara la existencia de 608.101 extremeños residiendo fuera de su tierra de origen.

Indudablemente, tan generosa presencia extremeña dentro y fuera de España ha constituido una especie de reserva poblacional que ha servido para alimentar un flujo de retorno que adquirió una dimensión significativa hacia mediados de los años setenta y que entre 1991 y 2009 ha supuesto el regreso a Extremadura de 93.444 antiguos emigrantes.

Ésta es la inmigración de retorno que pretende analizarse en el presente artículo. La constatación de que este flujo inmigratorio manifiesta una marcada sensibilidad ante los cambios de coyuntura económica, invita a formular la hipótesis de que la actual situación de crisis en que se mueve la economía mundial debe de estar propiciando la aparición de cambios cuantitativos y estructurales en el retorno de antiguos emigrantes hacia Extremadura. En función de estos planteamientos, se analizará el volumen y estructura de la inmigración de retorno desde finales de los años ochenta, se significarán las transformaciones que se han experimentado tanto en su volumen como en sus rasgos estructurales, y se evaluará la incidencia que ejercen las variables procedencia y destino en las características de dicha corriente migratoria.

La fuente utilizada para la realización de este artículo ha sido la Estadística de Variaciones Residenciales que «... se elabora por el Instituto Nacional de Estadística básicamente a partir de la explotación de la información relativa a las altas y bajas por cambios de residencia registradas en los Padrones municipales $»^{2}$. Esta Estadística viene realizándose desde 1966, fecha ésta desde la que ha experimentado importantes cambios que han afectado tanto a su cobertura como a sus contenidos y medios de difusión. Si bien en la actualidad están disponibles ficheros de microdatos de la EVR desde 1988 a 2010, lo cierto es que anteriormente sólo podía accederse libremente a los posteriores al año 1998. No obstante, el INE ofrecía la

2 INE, www.ine.es. Estadística de Variaciones Residenciales. Metodología. 
posibilidad de acceder a esta información mediante la comercialización de los registros anonimizados de dichos ficheros. Por este motivo, los datos utilizados en el análisis del período 1988 a $2000^{3}$ se han obtenido por este procedimiento, en tanto que los pertenecientes a la etapa 2001-2009 se han conseguido de forma gratuita accediendo a los ficheros de microdatos de la Encuesta de Variaciones Residenciales que ofrece el INE en su página web.

Conviene advertir que, aunque la Estadística de Variaciones Residenciales es la fuente más fiable y completa para el análisis de las migraciones, adolece de una serie de deficiencias que, en el caso que nos ocupa y entre otras consecuencias, sólo permite una valoración a la baja del volumen de retornados. El motivo fundamental de este subregistro se debe a que hasta 1996, fecha en que se establece un nuevo sistema de gestión de los Padrones municipales basado en su informatización y coordinación por parte del INE, en los años en que se realizaba un recuento oficial de población, los retornados podían sustituir la tramitación del alta padronal por su inscripción directa en la hoja padronal, escapando de este modo al concepto de variación residencial.

Por otra parte, es frecuente que los antiguos emigrantes regresen con cónyuges o hijos no nacidos en Extremadura y que, por lo tanto, debieran considerarse como integrantes de esta corriente de retorno. La estructura de la Estadística de Variaciones Residenciales, no obstante, no permite su cuantificación, ya que aparecen integrados en el flujo inmigratorio general. Pese a todo, la fuente utilizada permite el análisis de las variables sexo, edad, provincia y municipio de nacimiento, origen y destino de los desplazamientos, y tamaño de los municipios de origen de las altas y destino de las bajas. Al mismo tiempo, la Estadística de Variaciones Residenciales facilita el análisis de las migraciones a nivel municipal, ofreciendo la posibilidad de contrastar la dinámica migratoria a distintas escalas y valorar su alcance sobre el comportamiento demográfico de ámbitos territoriales diferenciados.

Para valorar la intensidad del retorno nacional durante el período 2001-2009, se ha utilizado el Padrón de Habitantes de 2005. A partir de él se ha podido valorar el volumen de emigrantes extremeños que reside en cada una de las Comunidades Autónomas y, de ese modo, calcular el porcentaje de retornados que, sobre dicho volumen, proporciona cada una de ellas.

\section{DEL RETORNO TRADICIONAL A LA CONSOLIDACIÓN DE NUEVOS PATRONES}

Hasta que el INE puso a disposición de los investigadores la Estadística de Variaciones Residenciales, el análisis del retorno requería la consulta directa y pormenorizada de las altas cursadas por los retornados en los municipios de destino. En trabajos anteriores (Pérez Díaz, 1990; Barrientos, Pérez y Rengifo, 1993) tuvimos ocasión de comprobar las alteraciones migratorias que se derivaron de la crisis económica que afectó a la economía mundial hacia mediados de los años setenta. Así, al agotamiento de la corriente emigratoria hacia Europa, se sumaron la retracción de la emigración interior hasta niveles inferiores a la mitad del período precedente, y la consolidación de una corriente de retorno de rasgos estructurales sensiblemente distintos a los del flujo de salidas.

3 Dicha información se adquirió con cargo al Proyecto de Investigación «Situación actual y perspectivas del retorno a Extremadura» financiado por el Consejo de Comunidades Extremeñas (Junta de Extremadura). El proyecto se desarrolló entre 2001 y 2003, y fue Investigador Principal el Dr. D. Antonio Pérez Díaz. 
El análisis detallado de las altas y bajas padronales cursadas en los municipios del Norte de la provincia de Cáceres, permitió comprobar los acusados contrastes existentes en la estructura por edad de los flujos de entrada y salida. El 55,8 por 100 de los emigrantes comarcales tenía una edad comprendida entre los 15 y los 29 años. Los inmigrantes, por el contrario, superaban los 65 años en casi el 77 por 100 de los casos.

Tabla 1

ESTRUCTURA POR EDAD DE LOS MIGRANTES TRASIERRA-GREDOS SUR (1976-80)

\begin{tabular}{|c|c|c|}
\hline Edad & Emigrantes & Inmigrantes \\
\hline $0-14$ & 21,7 & 2,4 \\
\hline $15-29$ & 55,8 & 0,3 \\
\hline $30-49$ & 21,0 & 3,8 \\
\hline $50-64$ & 1,3 & 16,6 \\
\hline$>65$ & 0,2 & 76,9 \\
\hline TOTAL & 100,0 & 100,0 \\
\hline
\end{tabular}

Fuente: Vázquez Pérez, A. (1985)

A tenor de estos datos, por tanto, el panorama migratorio extremeño de la segunda mitad de los años setenta estuvo presidido por la pervivencia de un flujo emigratorio protagonizado por jóvenes con menos de treinta años, y por la consolidación de un retorno de antiguos emigrantes que en casi el 77 por 100 de los casos habían alcanzado la edad de jubilación. Demográficamente, ambos aspectos contribuyeron de forma decisiva a un afianzamiento del proceso de envejecimiento regional. En términos económicos, la situación se resolvía en una pérdida de población joven que, como contrapartida, se compensaba con el regreso de personas mayores con escasa capacidad de ahorro, un potencial inversor muy endeble y unas necesidades asistenciales y sanitarias difíciles de atender por entonces en Extremadura (Barrientos, 1990).

Pero la década de los ochenta habría de caracterizarse por la consolidación de nuevos patrones migratorios. Por primera vez en más de un siglo, el balance migratorio regional arrojó saldo positivo en las dos provincias extremeñas, en el 59 por 100 de las comarcas y en el 48 por 100 de los municipios. Y si novedosa resultaba tal circunstancia, de insólitos habría que calificar los nuevos rasgos que configuraban la estructura del retorno. Según datos del trienio 1988-90, este retorno de extremeños apenas estaba integrado en un 13 por 100 por personas mayores de 65 años, mientras que en un 52,8 por 100 de los casos los retornados tenían edades comprendidas entre los 15 y los 44 años.

Tabla 2

ESTRUCTURA POR EDAD DEL RETORNO (1988-90)

\begin{tabular}{|c|c|r|}
\hline Edad & Total & \% \\
\hline $0-14$ & 1.636 & 12,0 \\
\hline $15-44$ & 7.208 & 52,8 \\
\hline $45-64$ & 3.035 & 22,2 \\
\hline$>65$ & 1.763 & 12,9 \\
\hline TOTAL & 13.642 & 100,0 \\
\hline
\end{tabular}

Fuente: INE, Microdatos de la EVR (Elab. Propia) 
Esta nueva situación no podía explicarse argumentando una mejora sustancial y generalizada de la economía extremeña sino, más bien, la difícil situación socio-económica de los centros industriales. Buena parte del retorno se produjo como consecuencia de la pérdida de empleo de los miembros adultos de la familia o, en otros casos, por las dificultades de los jóvenes para insertarse en el mercado laboral. No debe olvidarse que la mejora que experimentó la economía española en la segunda mitad de los ochenta resultó insuficiente para absorber la creciente demanda laboral que representaba la presencia en el mercado de las nutridas generaciones del baby boom postbélico (Puyol, 1997). Ni tampoco debe ignorarse que esta reactivación económica se centró prioritariamente en los subsectores de la construcción y la hostelería, caracterizados ambos por la temporalidad y la precariedad de los empleos, circunstancia ésta que se transmite a la movilidad poblacional dotándola de unos rasgos de provisionalidad ausentes en la corriente migratoria tradicional. De ahí, por tanto, que los factores de rechazo y atracción ejercieran un juego de fuerzas equilibradas y provocaran, con ello, la coexistencia de flujos contrarios. La emigración de jóvenes extremeños que aspiraban a mayores y mejores perspectivas laborales, coincidía con el retorno de quienes sufrían la insatisfacción o el fracaso del proyecto emigratorio poco antes emprendido.

En la última década del pasado siglo, Extremadura registró un total de 93.267 altas procedentes de otras comunidades autónomas y del extranjero, y registró 92.633 bajas padronales con idéntico destino, arrojando así un débil balance migratorio positivo de 634 individuos. Pese a que se trata de datos brutos, la primera consideración que requiere este volumen de desplazamientos es que las migraciones extremeñas no constituyen un fenómeno exangüe. Una movilidad que se cifra en 185.900 cambios de residencia en una región cuya población

Tabla 3

EVOLUCIÓN DEL RETORNO A EXTREMADURA

\begin{tabular}{|c|r|r|r|r|r|}
\hline Años & Retorno Total & $\begin{array}{c}\text { Retorno } \\
\text { Nacional }\end{array}$ & $\begin{array}{c}\text { \% Retorno } \\
\text { Nacional }\end{array}$ & $\begin{array}{c}\text { Retorno } \\
\text { Extranjero }\end{array}$ & $\begin{array}{c}\text { \% Retorno } \\
\text { Extranjero }\end{array}$ \\
\hline 1991 & 3.538 & 3.423 & 96,7 & 115 & 3,3 \\
\hline 1992 & 5.021 & 4.789 & 95,4 & 232 & 4,6 \\
\hline 1993 & 5.494 & 5.292 & 96,3 & 202 & 3,7 \\
\hline 1994 & 5.508 & 5.322 & 96,6 & 186 & 3,4 \\
\hline 1995 & 5.107 & 4.882 & 95,6 & 225 & 4,4 \\
\hline 1996 & 3.520 & 3.392 & 96,4 & 128 & 3,6 \\
\hline 1997 & 4.055 & 3.893 & 96,0 & 162 & 4,0 \\
\hline 1998 & 4.420 & 4.203 & 95,1 & 217 & 4,9 \\
\hline 1999 & 4.613 & 4.388 & 95,1 & 225 & 4,9 \\
\hline 2000 & 4.274 & 4.064 & 95,1 & 210 & 4,9 \\
\hline TOTAL & 45.550 & 43.648 & 95,8 & 1.902 & 4,2 \\
\hline
\end{tabular}

Fuente: INE, Microdatos de la EVR (Elaboración propia) 
se situó en 1.069.420 habitantes en el año 2000, está indicando la permanencia de unos flujos que condicionan sobremanera la evolución, las características estructurales y la distribución espacial de la población extremeña.

Este es el escenario en el que debe analizarse la inmigración de retorno a Extremadura. Entre los años 1991 y 2000, esta corriente ha supuesto el regreso de 45.550 antiguos emigrantes y, con frecuencia, la llegada de sus cónyuges e hijos nacidos fuera de esta región. Este volumen de retornados arroja un promedio anual de 4.555 regresos, cifra nada desdeñable tanto si se considera la debilidad poblacional de Extremadura como si se relaciona con el volumen total de entradas registradas en la región, en cuyo caso se alcanzaría una participación del 48,8 por 100 (tabla 4).

Hay que significar, no obstante, que esa media anual enmascara la existencia de acusadas fluctuaciones. Algunas de ellas tienen su origen en la fuente estadística manejada. Se trata del subregistro que se produce en el año censal de 1991 y en 1996, fecha de realización del Padrón Municipal de Habitantes.

Las restantes variaciones del volumen de retornados pueden obedecer a situaciones muy diversas, aunque básicamente se aprecia una superior afluencia de retornados en los períodos en los que la economía nacional acusa mayores dificultades en la generación y estabilidad de los empleos (Recaño y Cabré, 2003). De ahí, probablemente, los contrastes existentes entre los contingentes de retornados contabilizados en la primera y segunda mitad de los años noventa. Conviene añadir, a este respecto, que buena parte de los emigrantes extremeños de dicho período se empleaban en los subsectores de la construcción y la hostelería, muy sensibles ambos a los cambios de coyuntura económica. Es verosímil, en consecuencia, que las oscilaciones que se registraran en ambas actividades se transmitieran de forma inmediata a los flujos migratorios, determinando la existencia de ritmos alternantes en la emigración y el retorno.

En este mismo sentido, conviene matizar la evolución que ha venido experimentando el peso del retorno dentro de la corriente inmigratoria general. La representatividad del retorno dentro del flujo inmigratorio total no ha dejado de reducirse a lo largo de la década de los noventa, de modo que si hasta el año 1996, cinco de cada diez altas registradas en Extremadura eran cursadas por retornados, en el año 2000 la proporción bajó a cuatro de cada diez. Pero dicha situación no sólo se debió a un debilitamiento del retorno, sino también a un reforzamiento general de la corriente inmigratoria, tanto interior como extranjera.

En este período, Extremadura se acomodó al perfil inmigratorio que manifestaban otras muchas regiones españolas (Recaño, 2004), y lo hizo tanto en sus ciudades como en algunos de sus más significados territorios rurales. A primera vista, esta situación podría resultar contradictoria, pues cabría pensar que un aumento de la capacidad de atracción inmigratoria de Extremadura debiera reflejarse de igual modo en un incremento del regreso de sus antiguos emigrantes. No obstante, las entrevistas y encuestas realizadas a la población retornada permitieron comprobar que la decisión del regreso es mucho más meditada que la de la emigración. Retornar es volver a emigrar y, cuanto más tiempo haya transcurrido desde la salida, mayores son los inconvenientes con que tropieza el regreso y la desconfianza y el recelo que genera en sus potenciales protagonistas. 
Tabla 4

PARTICIPACIÓN DEL RETORNO SOBRE LA INMIGRACIÓN TOTAL

\begin{tabular}{|c|c|c|c|}
\hline Años & Retorno Total & Inmigración Total & \% Retorno \\
\hline 1991 & 3.538 & 6.229 & 56,8 \\
\hline 1992 & 5.021 & 9.120 & 55,1 \\
\hline 1993 & 5.494 & 10.058 & 54,6 \\
\hline 1994 & 5.508 & 10.530 & 52,3 \\
\hline 1995 & 5.107 & 9.856 & 51,8 \\
\hline 1996 & 3.520 & 6.971 & 50,5 \\
\hline 1997 & 4.055 & 8.630 & 47,0 \\
\hline 1998 & 4.420 & 10.078 & 43,9 \\
\hline 1999 & 4.613 & 11.280 & 40,9 \\
\hline 2000 & 4.274 & 10.515 & 40,6 \\
\hline TOTAL & 45.550 & 93.267 & 48,8 \\
\hline
\end{tabular}

Fuente: INE, Microdatos de la EVR (Elaboración propia)

El retorno es, en buena parte de los casos, el fruto de una decisión compartida con el resto de la familia, tanto si es porque parte de ella decide permanecer en el lugar de residencia, como si es porque el desplazamiento les afecta a todos. Indudablemente estas circunstancias merman flexibilidad al retorno y le impiden reaccionar ante determinadas situaciones con la misma fluidez que la inmigración convencional (Izquierdo y Álvarez, 1997).

Junto a estas consideraciones relativas a la evolución del número de regresos, debe reseñarse el claro predominio que corresponde al retorno de origen nacional, con una media del 95,8 por 100 y una participación anual que en ningún caso se ha situado por debajo del 95 por 100. Obviamente, tal desproporción no hace sino reflejar la diferente intensidad que adquirieron la emigración interior y la exterior y, en consecuencia, la diferente dimensión que tienen las colonias de extremeños que residen, respectivamente, dentro y fuera del territorio nacional. Podría añadirse, a este respecto, que el retorno procedente del extranjero alcanzó sus más elevadas cotas hacia la segunda mitad de los años setenta y primera de los ochenta. Fue entonces cuando se produjeron los embates más duros de la crisis económica y, coincidiendo con ellos, los duros reajustes de plantilla y el cierre de empresas, circunstancias todas que determinaron la práctica paralización de la emigración a Europa y reanimaron el regreso desde el extranjero (Mertins, 1983). El retorno interior, sin embargo, además de nutrirse de un colectivo mucho más amplio, no ha dejado de beneficiarse de la mayor movilidad migratoria que se deriva de la temporalidad y la precariedad del mercado laboral.

En lo que respecta a sus rasgos estructurales, el retorno registrado a lo largo de la década de los noventa aparece caracterizado por la existencia de un marcado equilibrio en la distribución por sexos y por el predominio de retornados con menos de 45 años. En líneas generales, estos rasgos responden a los mismos patrones que lograron consolidarse en la década 


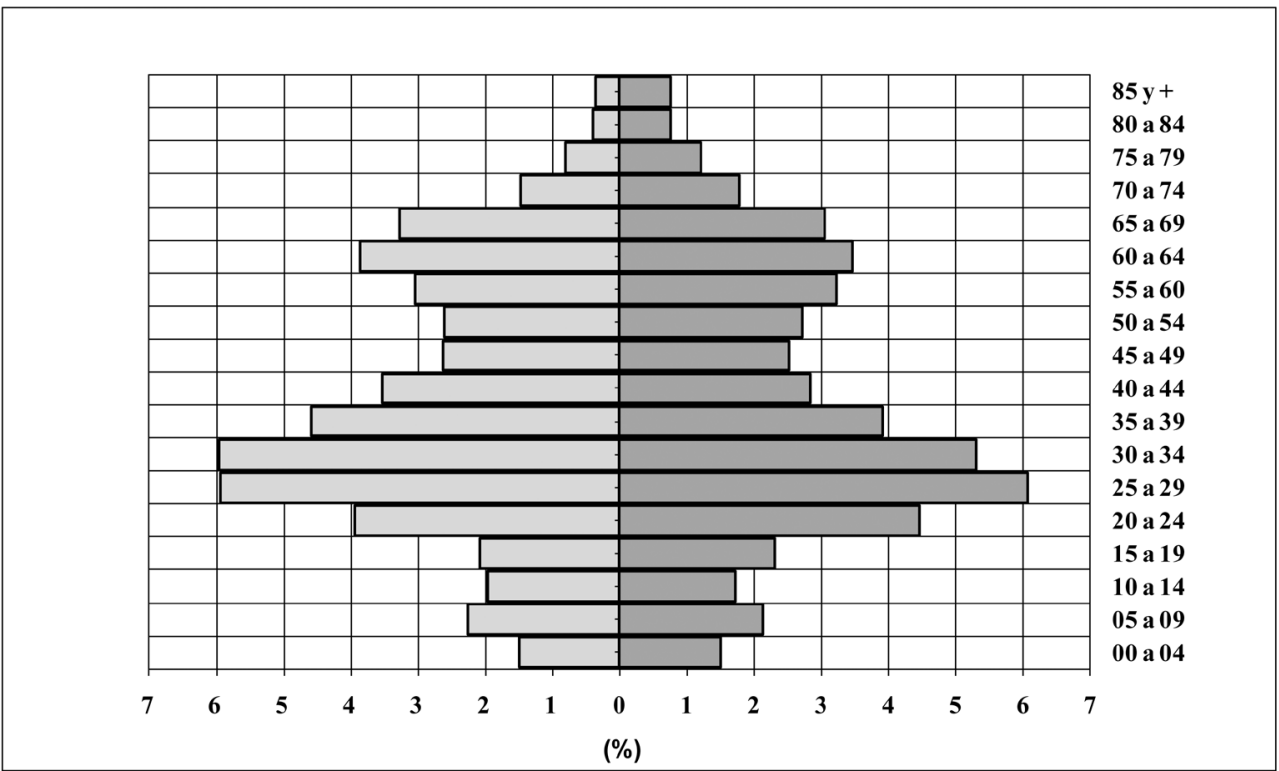

Fuente: INE, Microdatos de la EVR (Elaboración propia)

de los ochenta, por cuanto que apenas se aprecian unas variaciones mínimas en relación con aquel período.

En su aspecto general, la pirámide de edades del retorno destaca por la equilibrada distribución entre hombres y mujeres que se produce a casi todas las edades, de forma que en el cómputo total apenas destacan mínimamente los hombres $(50,2$ por 100) y lo hacen justamente con la misma proporción que consiguen en la emigración extrarregional (Pérez y Barrientos, 2006). Al margen de esta circunstancia, la pirámide se resuelve en la existencia de una base muy alta y estrecha que, a modo de pilar, sustenta una estructura que adquiere su máximo desarrollo a la altura correspondiente a las cohortes que tienen entre 20 y 39 años, que se retrae sensiblemente entre los 40 y 54 años y que vuelve a mostrar un engrosamiento considerable entre los 55 y 69 años, con especial relevancia en el lado de los hombres.

Para una correcta interpretación de la figura 1 se ha de insistir nuevamente en un hecho ya comentado. El que los jóvenes menores de 15 años sólo constituyan el 11,4 por 100 sobre el total de retornados no debe interpretarse como un reflejo fiel de la escasa incidencia que tiene el regreso de matrimonios jóvenes con hijos pequeños. En gran medida, dicha circunstancia se debe a que tales hijos han nacido fuera de Extremadura y, por tanto, no pueden contabilizarse como retornados sino como inmigrantes. Es verosímil, por tanto, la existencia de una base más amplia y, por ende, más ajustada en sus dimensiones a la abultada participación que corresponde a los retornados con edad coincidente con la de los potenciales padres de estos niños y adolescentes. 
Tabla 5

CARACTERISTICAS DEL RETORNO (1991-2000)

\begin{tabular}{|l|r|}
\hline \multicolumn{1}{|c|}{ Características } & \multicolumn{1}{c|}{$\%$} \\
\hline Hombres & 50,2 \\
\hline Mujeres & 49,8 \\
\hline Menos de 15 años & 11,4 \\
\hline Entre 16 y 44 & 51,1 \\
\hline Entre 45 y 64 años & 24,2 \\
\hline Más de 64 años & 13,3 \\
\hline Índice de Envejecimiento & 116,7 \\
\hline
\end{tabular}

Fuente: INE, Microdatos de la EVR (Elaboración propia)

En conclusión, pues, desde la segunda mitad de los años ochenta la inmigración de retorno a Extremadura insinúa la configuración de un nuevo comportamiento, sensiblemente distinto al precedente, en el que se significa la participación creciente de población joven y adulta joven, hasta situarse por encima del contingente de población mayor de sesenta y cinco años. A partir de esta fecha y hasta finales del siglo XX, este retorno de extremeños afecta a una media de unas 4.500 personas al año, aunque su representatividad dentro del flujo inmigratorio total mantiene una tendencia descendente debido a la convergencia de dos circunstancias distintas: de un lado, el retraimiento del propio retorno; de otro lado, el reforzamiento de la inmigración, tanto nacional como extranjera. En todo caso, la persistencia de rasgos estructurales similares a los de la etapa anterior, permiten confirmar la consolidación de nuevos patrones en la inmigración de retorno a Extremadura.

\section{CRISIS ECONÓMICA Y RETORNO EN LOS INICIOS DEL SIGLO XXI}

El panorama migratorio que muestra la región extremeña en los primeros años del presente siglo, sugiere la existencia de algunos cambios de interés en relación con el período anteriormente analizado. Debe significarse, en primer lugar, el incremento que ha experimentado la corriente inmigratoria, que ha pasado de las 93.267 altas registradas en el periodo 1990-2000 a las 154.748 contabilizadas entre 2001 y 2009. De igual modo, ha de destacarse el aumento del flujo emigratorio, tanto hacia otras regiones españolas como hacia el extranjero, de modo que se ha pasado de las 92.633 bajas del primer período a las 127.853 de este otro más reciente. Finalmente, no debe pasar desapercibido el crecimiento de la movilidad interna, que se ha situado en la cota de los 105.297 cambios de residencia entre municipios distintos, superando con creces los 88.024 cambios de la etapa anterior. Teniendo en cuenta que la duración de los dos períodos comparados es distinta, el flujo inmigratorio se habría incrementado en un 84,4 por 100 , el correspondiente a la corriente emigratoria lo habría hecho en un 53,4 por 100 y las migraciones interiores habrían crecido en un 32,9 por 100 . Habida cuenta de la debilidad demográfica de Extremadura, no cabe duda de que esta intensificación de los flujos migratorios está ejerciendo mayor influencia que la dinámica vegetativa en la estabilización poblacional de la región, máxime si se considera que el balance 
migratorio arroja un saldo favorable de 26.895 individuos, lo que implica que se ha multiplicado por más de 42 en relación con la década final del siglo XX.

Tabla 6

EVOLUCIÓN DEL RETORNO A EXTREMADURA (2001-2009)

\begin{tabular}{|c|c|c|c|c|c|}
\hline Años & Retorno Total & $\begin{array}{c}\text { Retorno } \\
\text { Nacional }\end{array}$ & $\begin{array}{c}\text { \% Retorno } \\
\text { Nacional }\end{array}$ & $\begin{array}{c}\text { Retorno } \\
\text { Extranjero }\end{array}$ & $\begin{array}{c}\text { \% Retorno } \\
\text { Extranjero }\end{array}$ \\
\hline 2001 & 3.714 & 3.592 & 96,7 & 122 & 3,3 \\
\hline 2002 & 4.873 & 4.714 & 96,7 & 159 & 3,3 \\
\hline 2003 & 5.712 & 5.502 & 96,3 & 210 & 3,7 \\
\hline 2004 & 5.598 & 5.360 & 95,7 & 238 & 4,3 \\
\hline 2005 & 5.122 & 4.917 & 96,0 & 205 & 4,0 \\
\hline 2006 & 5.929 & 5.692 & 96,0 & 237 & 4,0 \\
\hline 2007 & 5.990 & 5.705 & 95,2 & 285 & 4,8 \\
\hline 2008 & 5.395 & 5.185 & 96,1 & 210 & 3,9 \\
\hline 2009 & 5.561 & 5.398 & 97,1 & 163 & 2,9 \\
\hline TOTAL & 47.894 & 46.065 & 96,2 & 1.829 & 3,8 \\
\hline
\end{tabular}

Fuente: INE, Microdatos de la EVR (Elaboración propia)

En este contexto general, la inmigración de retorno también ha logrado incrementarse hasta alcanzar la cifra de 47.894 altas, lo que representa un aumento medio del 16,8 por 100 en relación con el volumen registrado a finales del pasado siglo.

En lo que respecta a su evolución, debe insistirse nuevamente en que algunas de las fluctuaciones interanuales tienen su origen en la peculiaridad de la Estadística de Variaciones Residenciales que, tal como se advierte en 2001, conduce a un subregistro en los años censales. No obstante, podría significarse una cierta disimetría entre el primer quinquenio del período y los cuatro años finales, de modo que se apreciaría un aumento en el promedio anual de retornados al pasar de los 5.003,8 de 2001-2005 a los 5.718,8 de 2006-2009. Aunque pudiera parecer una maniobra forzada, esta distinción permitiría relacionar el comportamiento más reciente del retorno con la agudización de la crisis económica. Ciertamente, habrá que aguardar hasta disponer de nuevos datos para poder confirmar dicha hipótesis, pero en principio parece verosímil un aumento del flujo de retornados propiciado por la destrucción de empleo que ha padecido el sector de la construcción, principal destino laboral de la emigración extremeña de las últimas décadas.

No obstante, la estructura por edad de los retornados, también permitiría insistir en idéntica dirección, de tal modo que podría colegirse que esa misma destrucción de empleo urbano que serviría para explicar el crecimiento del volumen de retornados, estaría propiciando igualmente un mayor retorno de varones y un rejuvenecimiento del colectivo (Tabla 7). En cualquier caso, debe insistirse en la necesidad de disponer de mayor perspectiva para poder determinar que no se trata sólo de fluctuaciones inherentes a la propia volatilidad del fenómeno estudiado.

De hecho, la estructura por edades correspondiente a la totalidad del período, continúa ajustándose a los patrones que lograron consolidarse en la segunda mitad de los ochenta, de 
Tabla 7

ESTRUCTURA POR SEXO Y EDAD DEL RETORNO

\begin{tabular}{|l|r|r|}
\hline \multicolumn{1}{|c|}{ Características } & $\mathbf{2 0 0 1 - 2 0 0 5}$ & $\mathbf{2 0 0 6 - 2 0 0 9}$ \\
\hline Hombres & 50,5 & 51,5 \\
\hline Mujeres & 49,5 & 48,5 \\
\hline Menos de 15 años & 7,7 & 8,9 \\
\hline Entre 16 y 44 & 45,4 & 46,5 \\
\hline Entre 45 y 64 años & 25,8 & 26,2 \\
\hline Más de 64 años & 21,1 & 18,4 \\
\hline Índice de Envejecimiento & 274,2 & 206,5 \\
\hline
\end{tabular}

Fuente: INE, Microdatos de la EVR (Elaboración propia)

modo que sigue siendo significativo el equilibrio en la distribución por sexos y el predominio de retornados con edades inferiores a los 45 años.

No obstante, la procedencia del retorno genera variaciones significativas en su estructura. Las pirámides de edades del retorno nacional y extranjero presentan marcadas diferencias. Como es obvio, dada la acusada representatividad del retorno nacional, la primera apenas difiere de la estructura correspondiente al conjunto. La segunda, por el contrario, destaca por su morfología irregular y por la profusión de disimetrías en la distribución por sexos de la mayoría de las cohortes.

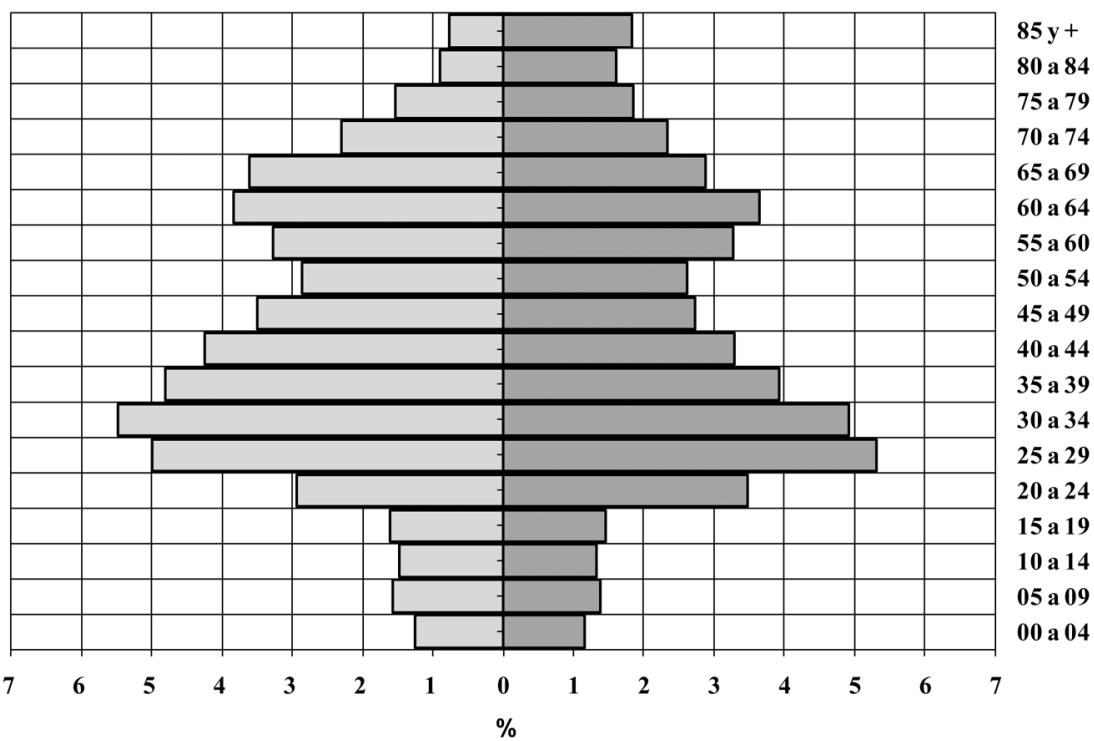

Fuente: INE, Microdatos de la EVR (Elaboración propia) 
El retorno nacional presenta una distribución por sexos equitativa tanto a nivel de conjunto como en prácticamente todas las cohortes. Es cierto que en las edades activas se aprecia un ligero predominio masculino, de igual modo que son más abundantes las mujeres en las edades más avanzadas. No obstante, ninguna de dichas circunstancias es suficiente para dudar del efecto regenerador que debe estar produciendo el retorno nacional sobre la demografía extremeña. Además de este equilibrio de géneros, el regreso de emigrantes desde otras regiones españolas implica un aporte de población que en el 54,6 por 100 de los casos tiene menos de 45 años, circunstancia ésta que debe ser valorada desde perspectivas optimistas en función de sus innegables consecuencias demográficas, sociales y económicas. Por el contrario, sólo un 19,6 por 100 de los retornados superan la edad de 65 años, una proporción ésta que se sitúa en cotas similares a la participación que alcanzan los mayores en el conjunto de la población extremeña (19,1 por 100).

Y aunque fuera muy superior, tal circunstancia seguiría mereciendo una valoración positiva tanto en el plano afectivo como en el sociolaboral. En el primer caso, por cuanto que representa la vuelta a casa de quienes hace tiempo se vieron forzados a abandonar su tierra y los suyos por la escasez de oportunidades y la falta de perspectivas de futuro. En el segundo, porque los mayores de hoy disponen de una capacidad física, intelectual y económica muy superior a la de hace unos pocos años, porque aún pueden aportar mucho en los municipios en los que nacieron y porque la satisfacción de sus demandas y de sus necesidades deben convertirse en nuevos yacimientos de empleo para los activos extremeños (Abellán y Puga, 2005).

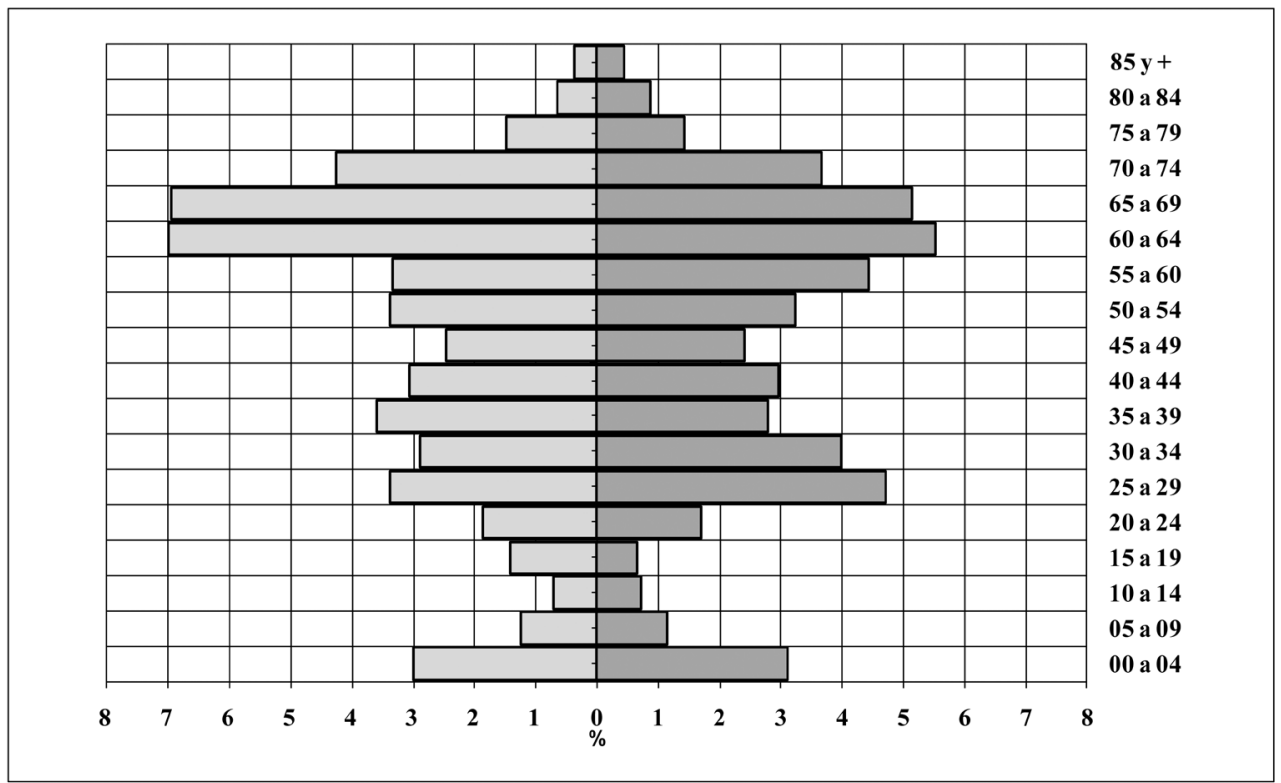

Fuente: INE, Microdatos de la EVR (Elaboración propia) 
Diferente es el caso del retorno procedente del extranjero, ya que tanto el desequilibrio en la distribución por sexos como sus rasgos de envejecimiento resultan muy acentuados. Sin embargo, debe recordarse que tan sólo está integrado por 1.829 personas, con lo que en ningún caso es posible atribuirle un nivel de incidencia mínimamente comparable al del retorno nacional. En cualquier caso, su pirámide de edades destaca por una morfología invertida en la que la endeblez de la base contrasta con la hipertrofia de las cohortes comprendidas entre los 60 y 75 años.

En esta ocasión, los retornados con menos de 15 años representan un 10 por 100 y los jóvenes con edades comprendidas entre los 20 y 24 años, un 3,6 por 100. Tan exigua representatividad obedece, de un lado, al marcado envejecimiento demográfico del colectivo extremeño en el extranjero y, en consecuencia, a su débil natalidad. Pero, por otro lado, no debe ignorarse el rechazo de los jóvenes a retornar, su deseo de permanecer en el lugar en el que han crecido, en el que se han educado y en el que han desarrollado su entorno afectivo y social. Esta es la razón por la que muchos emigrantes en el extranjero deben posponer su regreso hasta el momento en que sus hijos logran una independencia económica y deciden formar su propio hogar. Ciertamente, el contexto familiar, social y laboral del retorno extranjero difiere sustancialmente del nacional. En aquél los obstáculos son más frecuentes y variados, y la decisión del regreso debe ser más ponderada y consensuada en el ámbito de la familia.

La pirámide de edades presenta una cierta proporcionalidad en las cohortes de 35 a 55 años, aunque no escasean las irregularidades debidas, probablemente, a la escasa dimensión del retorno exterior y a las consiguientes dificultades para detectar pautas comunes y significadas. De ahí que su aspecto más llamativo se deba al sobredimensionamiento de las cohortes masculinas que tienen entre 60 y 70 años, un hecho que sigue reflejando, aunque sólo parcialmente, el protagonismo masculino que caracterizó a la emigración continental.

Aún así, es necesario significar que esta corriente de retorno se nutre prioritariamente de extremeños que emigraron con su familia o que tuvieron la oportunidad de reagruparse en el extranjero algún tiempo después de su salida. Es en estos casos cuando el regreso se retrasa hasta el momento en que se consolidan los derechos sociolaborales en las mejores condiciones económicas o cuando, como antes se indicaba, los hijos deciden su emancipación. Lógicamente, una vez conseguido este objetivo, el retorno se efectúa en compañía del cónyuge y, si es posible, en la de los hijos. Este es el motivo por el que, a pesar del marcado saliente que se produce en el lado de los hombres, existe mayor proporcionalidad en la distribución por sexos que en el retorno registrado en la segunda mitad de los setenta, cuando estuvo protagonizado por los extremeños que tiempo atrás habían emigrado hacia Europa sin la compañía de sus familiares.

En conclusión, pues, el retorno a Extremadura aparece constituido por dos corrientes bien distintas tanto en su volumen como en su composición: la interna, con un caudal anual que supera ligeramente las 5.118 personas y en la que destacan el equilibrio entre hombres y mujeres y el marcado predominio de la población adulta joven; y la exterior, sensiblemente más debilitada (203,2 retornados/año) en la que el protagonismo recae del lado de los hombres y los rasgos de envejecimiento demográfico, con un índice del 253,8 por 100, son palmarios. Una y otra se insertan en un contexto migratorio definido por causas idénticas y procesos de toma de decisiones semejantes. El origen de su diversidad, no obstante, se debe 
a la diferente dimensión y estructura sociodemográfica de las colonias de emigrantes extremeños que alimentan estos flujos. Por tal motivo, el lugar de procedencia seguirá marcando diferencias sensibles en el resto de características sociodemográficas del retorno.

De igual modo, el tamaño del municipio de destino también se erige en un factor condicionante de la estructura del retorno. Como se advierte en la tabla 8, la proporción de población joven se incrementa a medida que lo hace el tamaño del municipio de destino, de tal modo que los retornados con menos de 15 años sólo representan un 4,8 por 100 en el caso de los municipios con menos de 2.000 habitantes, en tanto que su participación se eleva hasta un 13,8 por 100 en las ciudades con más de 50.000 habitantes.

A diferencia de lo expuesto, el retorno de población vieja es sensiblemente superior, en términos relativos, en los municipios más pequeños, y reduce su participación a medida que aumenta el tamaño de los municipios de destino: sólo un 6,8 por 100 de los retornados que se han dirigido a las ciudades extremeñas con más de 50.000 habitantes, ha superado la edad de 65 años. Por el contrario, el colectivo de mayores ha supuesto el 27,7 por 100 del retorno que ha tenido como destino los municipios con menos de 2.000 habitantes.

Tabla 8

EDAD Y SEXO DE LOS RETORNADOS SEGÚN TAMAÑO DEL MUNICIPIO DE DESTINO

\begin{tabular}{|c|c|c|c|c|c|}
\hline \multirow{2}{*}{ Tamaño } & \multicolumn{3}{|c|}{ Edad } & \multicolumn{2}{|c|}{ Sexo } \\
\hline & $<15$ & $15-64$ & $>64$ & Varones & Mujeres \\
\hline$<2.000$ & 4,8 & 67,4 & 27,7 & 53,8 & 46,2 \\
\hline $2.000-5.000$ & 7,5 & 70,4 & 22,1 & 52,4 & 47,6 \\
\hline $5.001-10.000$ & 8,1 & 71,8 & 20,1 & 49,4 & 50,6 \\
\hline $10.001-50.000$ & 11,1 & 76,0 & 12,9 & 50,3 & 49,7 \\
\hline$>50.000$ & 13,8 & 79,4 & 6,8 & 49,9 & 50,1 \\
\hline
\end{tabular}

Fuente: INE, Microdatos de la EVR (Elaboración propia)

La distribución por sexos también manifiesta valores diferentes según este mismo parámetro. En los municipios con menos de 5.000 habitantes, el retorno tiene una componente predominantemente masculina, en tanto que existe un mayor equilibrio, cuando no un dominio femenino, si el retorno tiene su destino en municipios de mayor tamaño. En uno y otro caso, resulta ineludible entender este movimiento inmigratorio como una corriente heterogénea tanto en su vertiente estructural como en la espacial, hecho que a su vez remite a la existencia de motivaciones muy diferentes como factores explicativos del regreso a la región de origen.

Desagregando algunos de estos datos, debe significarse que el 56,4 por 100 de los retornados que tienen como destino los municipios extremeños con menos de 10.000 habitantes, superan los cuarenta y cinco años de edad, que sólo un 13,8 por 100 tienen menos de 24 años y que un 17,3 por 100 tienen más de setenta años, situándose el Índice de Envejecimiento en un proporción de 462,1 mayores de 65 años por cada cien menores de 15. En el caso de Cáceres, Badajoz y Mérida, las únicas ciudades de la región que superan el umbral de los 50.000 habitantes, los valores se sitúan en un 27,4 por 100 para los mayores de 45 años, un 24,8 por 100 para los menores de 24 años y sólo un 5,3 por 100 para los mayores de 70 años. 
En este caso, el Índice de Envejecimiento se sitúan en un 66,7 por 100, alejándose significativamente del correspondiente a los municipios más pequeños.

Ciertamente, no es difícil explicar estos contrastes. El retorno a las ciudades tiene una componente económica muy superior a la que pueda tener el regreso hacia los pequeños municipios extremeños. El regreso de población en edad activa, tanto si es motivado por factores positivos como si se debe a algún contratiempo laboral sufrido fuera de Extremadura, tiene la finalidad de encontrar un nuevo empleo, de iniciar una nueva actividad económica o reanudar la que se dejó al emigrar. Indudablemente, las ciudades ofrecen a estos retornados mayores posibilidades laborales y socioeconómicas que los pueblos. Estos últimos, sin embargo, resultan más atractivos para los retornados que han alcanzado la edad de jubilación o para quienes han accedido a la prejubilación. En este caso, son los lazos familiares y afectivos los que adquieren una relevancia especial al explicar el retorno. Prioritariamente, se vuelve al pueblo en el que se nació, con independencia de su tamaño, de su localización o de su situación y perspectivas socioeconómicas. Sólo en casos muy particulares, relacionados en general con la residencia de los familiares más próximos o con motivos de dependencia sociosanitaria, el retorno de los mayores tiene como destino un lugar diferente al de nacimiento y se advierte una preferencia hacia los núcleos de mayor tamaño.

En lo que respecta a los lugares de procedencia, tanto la corriente nacional como la extranjera muestran una marcada polarización geográfica, hecho éste que, indudablemente, no es más que un reflejo de la diferente capacidad de absorción de los focos de atracción de la emigración regional y del distinto volumen adquirido por las colonias de extremeños que han ido asentándose en ellos.

En lo que respecta al retorno nacional, la Comunidad Autónoma que mayor cantidad de retornados aportó a Extremadura entre 2001 y 2009 fue Madrid, con un total de 15.068 personas y una participación del 32,7 por 100. El hecho de que casi una tercera parte del retorno de emigrantes tenga origen en dicha región obedece a que éste ha sido uno de los destinos prioritarios de la emigración extremeña, tanto durante la etapa tradicional como en los últimos años. Habría que añadir además que la emigración reciente a Madrid, al menos hasta el recrudecimiento de la crisis económica, se ha nutrido prioritariamente de trabajadores de la construcción, circunstancia ésta que facilita el flujo y reflujo de extremeños en función de la oferta laboral que genera el sector y, en el lado opuesto, de la finalización de obras. No es de extrañar, por ello, que Madrid siga siendo el principal foco de atracción de la emigración extremeña actual al mismo tiempo que es el principal punto de origen del retorno regional.

Cataluña es, por motivos similares a los descritos para el caso de Madrid, la segunda emisora de retornados hacia Extremadura. Las 7.464 personas que han regresado desde aquella región representan un 16,2 por 100 sobre el total de retornados y, en gran medida, se corresponden con integrantes de aquella corriente migratoria tradicional que tuvo a Barcelona como uno de los principales centros de destino. No en vano, el 75,2 por 100 de los retornados desde Cataluña proceden de la provincia de Barcelona. A diferencia del foco madrileño que, como se ha dicho, mantiene aún una notable atracción sobre la emigración extremeña, el barcelonés ha perdido buena parte de su importancia tradicional a favor de otros centros menos distantes o con una oferta laboral centrada especialmente en los subsectores de la construcción y la hostelería. 
El País Vasco y Valencia, dos de los destinos tradicionales de la emigración extremeña, alcanzan una representatividad desigual como emisores de los retornados extremeños. Mientras que el primero mantiene una posición acorde con el volumen de extremeños que residen en su territorio, Valencia se descuelga hacia posiciones más rezagadas al haber cedido ante el empuje del retorno procedente de las regiones vecinas de Andalucía y Castilla-La Mancha.

Estos aparentes desajustes que se detectan entre el grado de atracción que ejercieron algunas Comunidades Autónomas sobre la emigración extremeña y su nivel de importancia como emisores del retorno, están motivados, entre otros, por factores como la distancia geográfica, el tiempo que media entre la emigración y el retorno, las características del flujo emigrante o el tamaño de la colonia extremeña que reside en los diferentes destinos.

Tabla 9

PROCEDENCIA E INTENSIDAD DEL RETORNO NACIONAL

\begin{tabular}{|l|r|r|r|r|}
\hline & \multicolumn{1}{|c|}{$\begin{array}{c}\text { Residentes } \\
\mathbf{2 0 0 5}\end{array}$} & $\begin{array}{c}\text { Retornados } \\
\mathbf{2 0 0 1 - 2 0 0 9}\end{array}$ & $\begin{array}{c}\text { Retornados/ } \\
\text { Residentes }\end{array}$ & \% Retorno total \\
\hline Andalucía & 63.311 & 5.033 & 7,9 & 10,9 \\
\hline Aragón & 8.404 & 596 & 7,1 & 1,3 \\
\hline Asturias & 7.271 & 250 & 3,4 & 0,5 \\
\hline Baleares & 13.468 & 3.851 & 28,6 & 8,4 \\
\hline Canarias & 5.435 & 2.225 & 40,9 & 4,8 \\
\hline Cantabria & 2.399 & 190 & 7,9 & 0,4 \\
\hline Castilla y León & 19.526 & 2.182 & 11,2 & 4,7 \\
\hline Castilla-La Mancha & 26.135 & 2.542 & 9,7 & 5,5 \\
\hline Cataluña & 148.957 & 7.464 & 5,0 & 16,2 \\
\hline C.Valenciana & 34.675 & 2.507 & 7,2 & 5,4 \\
\hline Galicia & 3.496 & 302 & 8,6 & 0,7 \\
\hline Madrid & 235.280 & 15.068 & 6,4 & 32,7 \\
\hline Murcia & 2.864 & 253 & 8,8 & 0,5 \\
\hline Navarra & 6.607 & 379 & 5,7 & 0,8 \\
\hline País Vasco & 63.500 & 2.781 & 4,4 & 6,0 \\
\hline Rioja (La) & 2.301 & 183 & 8,0 & 0,4 \\
\hline Ceuta & 464 & 131 & 28,2 & 0,3 \\
\hline Melilla & 337 & 128 & 38,0 & 0,3 \\
\hline TOTAL & 644.430 & 46.065 & 7,1 & 100,0 \\
\hline
\end{tabular}

Fuente: INE: Padrón de Habitantes y Microdatos de la EVR (Elaboración propia)

Para evaluar algunas de estas circunstancias, se ha procedido a calcular la intensidad del retorno, entendiendo por tal la relación existente entre el volumen de retornados procedente de una determinada Comunidad Autónoma y el número de extremeños que residían en ella con fecha 1 de enero de $2005^{4}$. De este modo, como puede apreciarse en la tabla 9, es posible distinguir al menos cuatro tipos diferentes de retorno.

4 Se ha utilizado esta fecha por entender que sirve de referencia media al período analizado (2001-2009) 
Un primer tipo está representado por los centros de destino tradicionales de la emigración extremeña, esencialmente Madrid, Cataluña y País Vasco, que disponen de una nutrida presencia de extremeños ${ }^{5}$, que ocupan las posiciones más destacadas como centros emisores de nuestros retornados, con un 5,49 por 100, pero que, sin embargo, muestran una muy baja intensidad, de tal modo que éste tan sólo alcanza a representar al 5,7 por 100 de los extremeños residentes o, lo que es lo mismo, de la población susceptible de retornar.

Probablemente, las razones que explican este aparente desajuste estén relacionadas con el mayor desarrollo económico de estas Comunidades Autónomas, con la mayor estabilidad de su mercado laboral, con el carácter tradicional y, por ende, con la permanencia más prolongada del colectivo extremeño en estos destinos y con el mayor envejecimiento de la colonia extremeña que reside en ellos, sobre todo en el caso del País Vasco y Cataluña.

El segundo patrón está representado por los focos emergentes de atracción de la emigración extremeña, especialmente Baleares y Canarias, en los cuales la presencia de extremeños es escasa, pero los niveles de representatividad como emisores de nuestros retornados alcanzan valores medios y los índices de intensidad resultan muy elevados. Como es bien conocido, los archipiélagos balear y canario se han convertido en los últimos años en un potente foco de atracción para los emigrantes extremeños, gracias a la oferta laboral que genera el turismo. Se trata de una emigración de carácter estacional que tiene una duración aproximada de seis meses y que ha venido adquiriendo una importancia creciente merced a que representa una clara oportunidad para complementar las rentas que se obtienen dentro de Extremadura el resto del año y para computar este trabajo como peonadas que han venido dando acceso a las percepciones del PER. Precisamente es este carácter estacional el que provoca la fuerte intensidad del retorno y el que da lugar a que también exista un marcado paralelismo entre las corrientes de emigración y retorno que tienen como destino y origen respectivo a Baleares y Canarias, circunstancia que no impide que la colonia de extremeños que está asentada fundamentalmente en Mallorca, tenga una dimensión significativa.

Un tercer caso estaría representado por Andalucía, Castilla-La Mancha y Castilla-León, en las que las razones de proximidad geográfica determinan la existencia de unos flujos de intercambio poblacional que, sin ser especialmente relevantes, sí han sido suficientes para generar una implantación relativamente significativa de extremeños en sus respectivos territorios. A consecuencia de ello, la proporción de retornados con dicha procedencia alcanza valores moderados, sobre todo en el caso de Andalucía, y los índices de intensidad se sitúan igualmente en niveles medios

Tampoco debe ignorarse la salida y la entrada de funcionarios y trabajadores cualificados que buscan en las capitales de las provincias vecinas unas condiciones socio-económicas que difícilmente pueden encontrarse en los municipios pequeños. Los casos de Sevilla y Salamanca son especialmente significativos en este sentido, ya que se trata de ciudades que tradicionalmente han atraído a parte del estudiantado universitario extremeño y, por tanto, a los que luego han sido licenciados y técnicos superiores que han desarrollado su actividad en dichas provincias. En ocasiones, el retorno de estas personas se produce cuando encuentran

5 Obsérvese que, de forma conjunta, estas tres Comunidades Autónomas acogen al 69,5 por 100 de los extremeños que residen fuera de su región de nacimiento. 


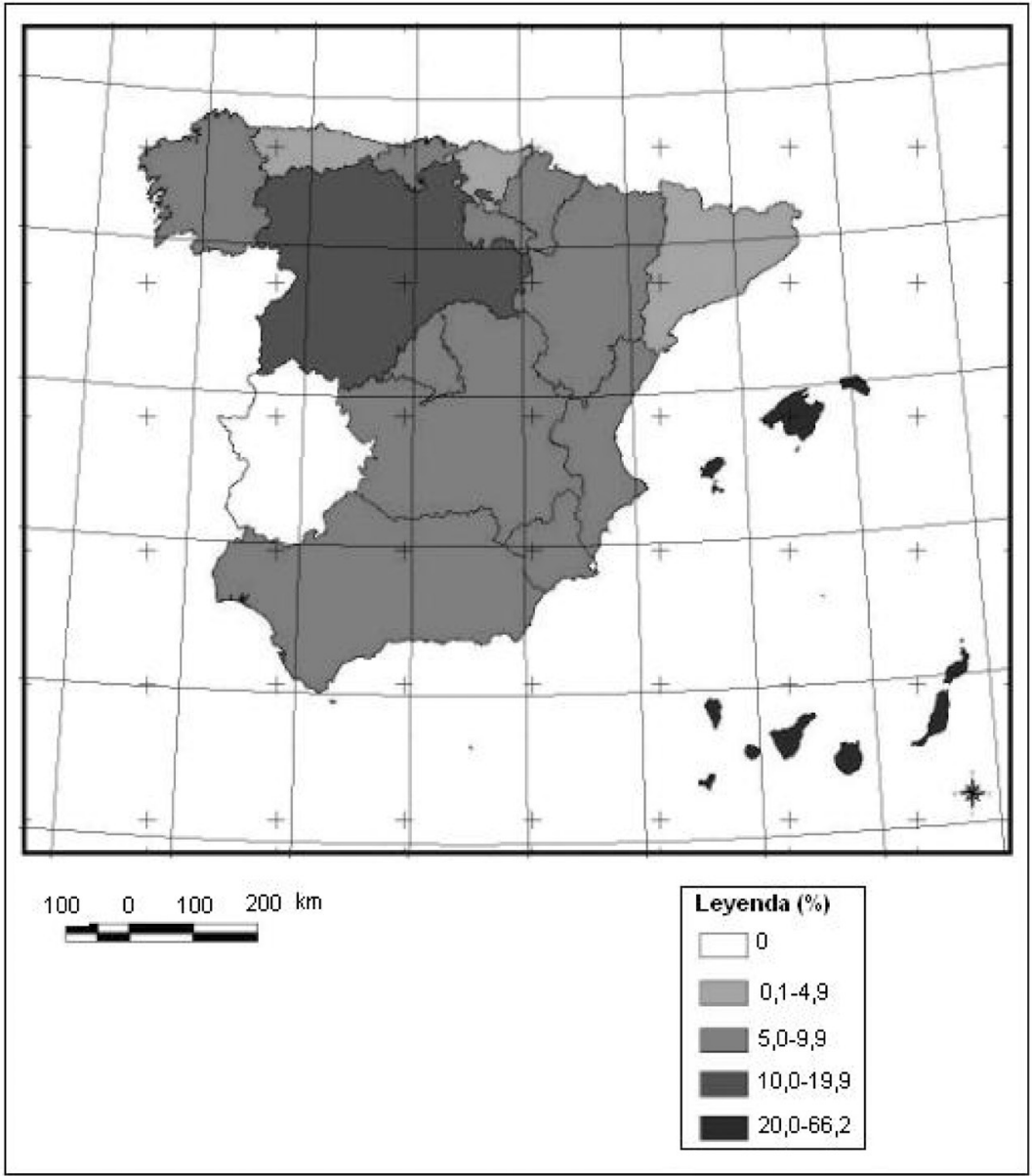

Fuente: INE: Padrón de Habitantes y Microdatos de la EVR (Elaboración propia)

una mejor oportunidad en Extremadura aunque, en muchos casos, éste no se efectúa hasta que se alcanza la edad de jubilación.

Finalmente, y más por su peculiaridad que por su significación estadística, podría distinguirse el ejemplo de Ceuta y Melilla, donde la presencia extremeña es exigua (apenas llega 0,1 por 100 en ambos casos), que ocupan las posiciones más rezagadas como emisores de los retornados ( 0,3 por 100$)$, pero en las que el retorno adquiere una muy elevada intensidad. En general, debe pensarse en un desplazamiento protagonizado especialmente por trabajadores de la Administración Pública que muy poco tiempo atrás emigraron hacia estas ciudades autonómicas y que han reemprendido el camino de regreso en cuanto han tenido una oportunidad para volver a Extremadura. Por razones bien conocidas, se trata de destinos muy poco atractivos y que, por ello, aparecen caracterizados por la transitoriedad de las estancias. 
Tabla 10

PROCEDENCIA DEL RETORNO EXTRANJERO

\begin{tabular}{|l|r|r|}
\hline \multicolumn{1}{|c|}{ País de procedencia } & Retornados & \multicolumn{1}{c|}{$\%$} \\
\hline Alemania & 396 & 21,7 \\
\hline Francia & 394 & 21,5 \\
\hline Suiza & 184 & 10,1 \\
\hline Portugal & 182 & 10,0 \\
\hline Países Bajos & 77 & 4,2 \\
\hline Andorra & 64 & 3,5 \\
\hline Reino Unido & 60 & 3,3 \\
\hline Bélgica & 57 & 3,1 \\
\hline Rumanía & 35 & 1,9 \\
\hline Italia & 21 & 1,1 \\
\hline Marruecos & 32 & 1,7 \\
\hline Argentina & 20 & 1,1 \\
\hline Brasil & 14 & 0,8 \\
\hline Venezuela & 11 & 0,6 \\
\hline Bolivia & 11 & 0,6 \\
\hline México & 10 & 0,5 \\
\hline Estados Unidos & 44 & 2,4 \\
\hline Australia & 7 & 0,4 \\
\hline Resto del mundo & 210 & 11,5 \\
\hline TOTAL & 1.829 & 100,0 \\
\hline
\end{tabular}

Fuente: INE, Microdatos de la EVR (Elaboración propia)

Para no abundar más en cuestiones que no revisten complejidad ni requieren más que una explicación mínima, podría concluirse significando que, como no podría ser de otro modo, los principales puntos de origen del retorno extremeño coinciden con los más destacados centros de destino de los emigrantes regionales. Algunos de ellos tienen un carácter tradicional, cual es el caso de Madrid, que continúa siendo un foco activo, Barcelona y las provincias vascas del litoral, focos actualmente muy debilitados. Algunos otros focos han experimentado un claro reforzamiento a lo largo del período analizado: es el caso de Sevilla, Toledo o Salamanca, donde la proximidad geográfica y las mayores posibilidades de desplazamiento juegan a favor de un intercambio poblacional más intenso; otros, en cambio, han surgido en los últimos años y se han convertido en potentes centros de atracción para los emigrantes estacionales y, en contrapartida, en puntos de origen importantes de retorno: sin duda, el ejemplo más significativo de este grupo lo constituyen las Islas Baleares, no obstante no deben ignorarse puntos de similar carácter como los de la costa catalana, el archipiélago canario o el litoral andaluz.

Por último, la procedencia de los retornados del extranjero apenas requiere matización alguna. Como puede apreciarse en la tabla 10, la mayor parte de las altas proceden de Ale- 
mania, Suiza, Francia y Portugal que, de manera conjunta, constituyen el origen del 63,3 por 100 del retorno que llega a Extremadura procedente del extranjero.

Luego, a bastante distancia, se sitúan los Países Bajos y a continuación, con valores similares, Andorra, Reino Unido y Bélgica. Estados Unidos, Argentina y Marruecos se sitúan como los puntos de origen extraeuropeo más importantes, aunque con escasa relevancia dentro del conjunto. En cualquier caso, debe significarse que entre 2001 y 2009 Extremadura ha recibido retornados procedentes de más de cincuenta países diferentes y de los cinco continentes. Ciertamente, algunas procedencias no alcanzan a tener más que un valor anecdótico. Pese a todo, bien pueden servir para reflexionar sobre el alcance geográfico que ha tenido la emigración extremeña y, merced a dicha dispersión, la variedad de situaciones que debe afrontar el extremeño que decide retornar a su tierra.

Y también debe animar dicha reflexión el hecho de que aún residan en otras regiones españolas $608.101^{6}$ personas nacidas en Extremadura, una cifra ésta que alcanza a representar el 54,9 por 100 del total de población censada en Extremadura en 2010, y un 63,9 por 100 de los nacidos y residentes en la región en dicha fecha. No son, ciertamente, datos irrelevantes. Por el contrario, reflejan con absoluta nitidez la envergadura del éxodo tradicional y la persistencia de la emigración en el momento actual. Pero, por encima de todo, tan voluminoso colectivo representa un potencial de retorno que puede y debe valorarse en una región tan demográficamente débil como Extremadura.

\section{CONCLUSIONES}

Los cambios de coyuntura que ha experimentado la economía desde mediados de los años setenta, han condicionado la evolución y el comportamiento de la inmigración de retorno a Extremadura, que ha venido experimentando en este tiempo una serie de cambios cuantitativos y estructurales. Desde la segunda mitad de los años ochenta, el retorno extremeño abandonó el patrón tradicional, caracterizado por el protagonismo indiscutible de los mayores de sesenta y cinco años, y se incorporó a nuevas pautas estructurales, que se mantuvieron a lo largo de los años noventa, caracterizadas por la participación creciente de población joven y adulta-joven hasta situarse claramente por encima del contingente de población vieja.

Durante la precedente etapa de bonanza económica, la región no sólo ha mostrado una capacidad creciente para atraer inmigrantes nacionales y extranjeros, sino que también consiguió atraer a un colectivo importante de antiguos emigrantes que aún se encontraban en edad activa, permitiendo con ello un rejuvenecimiento del retorno. Dicho cambio estructural continuaba respondiendo a los mismos patrones que se perfilaron en los años ochenta y se consolidaron a lo largo de la década de los noventa. No obstante, dicha caracterización general aparece sujeta a matizaciones vinculadas a variables como la procedencia de los retornados, el tamaño del municipio de destino, la distancia geográfica, el tiempo que media entre la emigración y el retorno, las características del flujo emigrante o el tamaño de la colonia extremeña que reside en los diferentes destinos.

La actual situación de crisis económica no ha interferido en dicho proceso de consolidación. Es más, al haber manifestado especial virulencia en el subsector de la construcción,

6 Actualización del Padrón de Habitantes a 1 de enero de 2010. 
una de las principales ocupaciones de los emigrantes extremeños desde mediados de los años ochenta, el cambio de coyuntura económica ha reactivado la corriente de retorno y ha forzado el regreso de población activa joven. Bien podría afirmarse, desde esta perspectiva, que en el primer decenio del siglo XXI han reverdecido algunos de los signos de dependencia que han caracterizado históricamente la economía regional. La dinámica espacial de los extremeños responde menos al empleo generado en la propia región, que a las fluctuaciones que experimenta la oferta laboral en el resto del territorio nacional. El optimismo que podría desprenderse de la existencia de un balance migratorio que ha arrojado saldos positivos entre 2007 y 2009, y al que contribuye de forma relevante la inmigración de retorno, se desvanece inexorablemente ante la escalada imparable del desempleo y la necesidad, tantas veces frustrada, de buscar empleo fuera de Extremadura. No es difícil columbrar una reactivación de la corriente emigratoria en un futuro escenario de recuperación económica. Tampoco lo es colegir la inmediatez y la intensidad de los desajustes que tal circunstancia provocaría en la demografía regional, en especial, sobre la de sus zonas rurales.

\section{BIBLIOGRAFÍA}

ABELLÁN, A. y PUGA, M.D. (2005): «Una España que envejece». Papeles de Economía Española, no $104,57-75$.

ARROYO, E. y MANCHADO, R. (1989): «Jaén: ¿Retorno de emigrantes?. II Jornadas sobre Población Española. Universitat de les Illes Balears, 459-478.

BARRIENTOS ALFAGEME, G. (1990): Geografía de Extremadura. Badajoz. Universitas Edit.

BARRIENTOS, G., PÉREZ, A. y RENGIFO, I. (1993): Migraciones y dependencia: Extremadura entre el éxodo y el retorno. Mérida. Junta de Extremadura- $\mathrm{M}^{\mathrm{O}}$ de Asuntos Sociales.

CASTILlO, J. (1980): «Emigrantes españoles: la hora del retorno». Papeles de Economía Española, $\mathrm{n}^{\circ}$ 4, 69-93.

CAYETANO ROSADO, M. (1996): Emigración asistida a Europa de la provincia de Badajoz durante el desarrollismo español (1961-75). Badajoz. Caja de Ahorros de Badajoz.

CAZORLA PÉREZ, J. (1981): Emigración y retorno. Una perspectiva europea. Madrid. Instituto Español de Emigración-M ${ }^{\circ}$ de Trabajo y Seguridad Social.

DELGADO, G. y ASCANIO, C. (1996): «El retorno reciente de emigrantes canarios: 1986 a 1993». Vegueta, n $^{\circ} 2,263-273$.

EGEA, C. y NIETO, J.A. (2001): «El retorno a la provincia de Jaén de emigrantes jubilados» Scripta Nova, núm. 94, 1 de agosto de 2001. Universidad de Barcelona. Disponible en http:// www.ub.edu/geocrit/sn-94-56.htm

GARCÍA COLL,A. (2005): «Migraciones interiores y transformaciones territoriales».Papeles de Economía Española, $\mathrm{n}^{\circ} 104,76-91$.

IZQUIERDO ESCRIBANO, A. y ÁLVAREZ SILVAR, G. (Coords.) (1997): Políticas de retorno de emigrantes. A Coruña: Universidade de A Coruña.

MERTINS, G. (1983): «El retorno de los emigrantes españoles en Europa: modelo de distribución espacial y conducta de inversión en España». Comentario sociológico, 31-32, 119-136. 
PASCUAL DE SANS, A. (1983): «Los movimientos migratorios de retorno. Significación y perspectivas». Documents d'Analisi Geográfica, $\mathrm{n}^{\circ}$ 3, 47-69.

PÉREZ DÍAZ, A. (1988): «Extremadura entre la emigración y el retorno». Alcántara, nº 3 y 4, 255-284.

PÉREZ DÍAZ, A. (1990): «Cambios estructurales en los movimientos migratorios extremeños» en Análisis del desarrollo de la población española en el período 1970-1986. Madrid. Edit. Síntesis, 138-144.

PÉREZ DÍAZ, A. (1997): Cara y cruz del temporerismo en Extremadura. Mérida. Junta de Extremadura.

PUJADAS, L., GARCÍA, A. y PUGA, D. (1995): «Migraciones interiores en España. Tendencias recientes y perspectivas de futuro (1971-2001)». Revista de Geografía. Vol. XXIX, n ${ }^{\circ} 3$.

PUYOL, R. (Ed.) (1997): Dinámica de la población en España. Cambios demográficos en el último cuarto del siglo XX. Madrid. Síntesis.

RECAÑO VALVERDE, J.(2004): «Las migraciones internas de retorno en España durante la primera mitad de la década de los noventa: implicaciones demográficas y territoriales. Scripta Nova, vol. VIII, núm. 157, 15 de enero de 2004. Universidad de Barcelona. Disponible en http:// www.ub.edu/geocrit/sn/sn-157.htm

RECAÑO, J. y CABRÉ, A. (2003): «Migraciones interregionales y ciclos económicos en España (1988-2001). Papeles de Geografía, n 185, 179-197.

ROMERO, J. y ALBERTOS, J.M. (1993): «Retorno al Sur, desconcentración metropolitana y nuevos flujos migratorios en España». REIS, $\mathrm{n}^{\circ}$ 63, 123-144.

VALADÉS SIERRA, J.M. (1994): Encuesta sobre la situación socio-económica y tendencia al retorno de los emigrados extremeños residentes en otras autonomías españolas. Mérida. Consejo de Comunidades Extremeñas. Junta de Extremadura.

VÁZQUEZ PÉREZ, A. (1985): Estudio de la ZAM Trasierra-Gredos Sur. 
